\title{
PNEUMATIC CONTACTLESS FEEDER FOR MICROASSEMBLY
}

\author{
Michele Turitto ${ }^{1}$, Dr. Yves-André Chapius ${ }^{2}$ and Dr. Svetan Ratchev ${ }^{3}$ \\ ${ }_{1,3}$ School of Mechanical, Materials and Manufacturing Engineering, University of

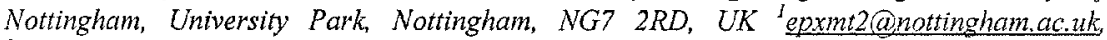 \\ ${ }^{3}$ svetan.ratchev@nottingham.ac.uk \\ ${ }^{2}$ Institute of Industrial Science, The University of Tokyo, 4-6-1 Komaba, Meguro-ku, Tokyo \\ 153-8505, Japan, chapuis@fuitita3. ils.u-tokyo.ac.jp
}

\begin{abstract}
The need of fully automated microassembly systems place specific functional requirements on the design and fabrication of critical elements such as grippers, sensors, manipulators and feeders. A new microfeeder design is proposed based on contactless pneumatic distributed manipulation. By cooperation of dynamically programmable microactuators, a number of feeding functions and even some elementary assembly operations can be achicved.
\end{abstract}

Key words: Microfeeding, Distributed manipulation, Pneumatic, Contactless, Microassembly

\section{INTRODUCTION}

The semiconductor industry over the last few decades has followed Moore's law: Dr. Gordon Moore in 1965 predicted the doubling of transistors in the same size of an IC every two years. The projections extend to the year 2012, at which time the smallest component of a device would have a linear dimension of $50 \mathrm{~nm}$ (1). The requirements in the IC industry have driven development of silicon-based micro and nano products. Silicon products are characterised by monolithic integration as all the process steps are integrated on a single substrate. This is an efficient but, at the same time, quite limiting approach as several different materials have to be combined with a silicon substrate to provide the high versatility and level of 
functionality needed in a variety of emergent microproducts (e.g. ink jet cartridges for printers, implantable drug delivery systems, chemical and biological sensors). Hence it is necessary to move from monolithic to hybrid integration so that the most appropriate material and fabrication techniques can be utilised.

Such new micromanufacturing requirements demand for an equally innovative approach to microassembly. If microproducts are to be produced in large volumes, novel materials, processes and production technologies have to be developed (2). This need cannot be addressed by simply transferring know-how from the macrodomain no matter how well established. Such a scaling down approach, although plausible, presents a number of flaws as it does not take into account the physical phenomena that emerge at the microscale (where surface-related forces become dominant over mass-related forces) and ignores issues like part fragility or part contamination.

In today's industries, the only alternative to automatic assembly, at least for the most critical operations, is manual assembly even if this can compromise the output in terms of time, cost and quality assurance. If high production volumes of microproducts have to be delivered in an efficient and reliable way, automatic assembly is the ultimate goal to which the efforts have to be aimed at. Figure 1 provides an economical justification for this last statement. It compares the product assembly cost for manual assembly and assembly on a highly flexible assembly cell for different batch sizes. The latter is more cost effective in all cases. The difference in total assembly cost is really significant for microsystems where manual assembly becomes too expensive due to the difficulty faced by a human operator when performing this type of operation. The same is true for the assembly of bigger products such as watches but in this case the advantage is less evident (3). Microassembly systems entail all the elements of an equivalent macroassembly system: grippers, sensors, manipulators, feeders and so forth.

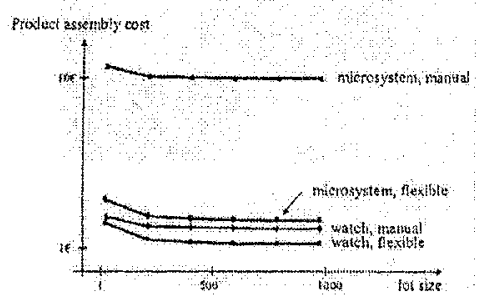

Figure 1. Flexible and manual assembly cost versus lot size for microsystems and watch components (3) 
There is a significant body of knowledge focused on microassembly gripping and actuating solutions whereas a crucial element such as feeding has not been sufficiently developed. In fact, it can be argued that part feeding is one of the most restrictive elements in achieving production flexibility and reconfigurability in microassembly systems. Flexibility in part feeding refers to the possibility of introducing new parts into the assembly system with minimal reconfiguration (4). A robust microfeeder has to fulfil this requirement and meet the constraints imposed by the level of precision.

This paper introduces a pneumatic microfeeder based on distributed manipulation fabricated by IC-compatible micromachining processes.

\section{MICROFEEDING}

Feeders have the function of presenting parts that were previously randomly oriented to an assembly station at the same position, with the correct orientation and the correct speed. In microassembly, distributed manipulation (figure 3 ) is a quite common approach for conveying microparts.

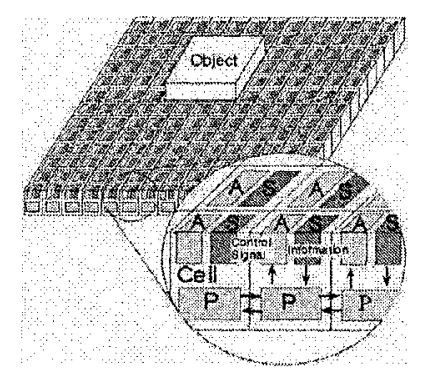

Figure 3. Distributed manipulation

It is based on arrays of tiny actuators where each is able to provide a simple motion. Even though the motion imparted by a single element is within a small range, it is possible to move objects over relatively long distances through the cooperation of a large number of microactuators. In this way a complex motion task is decomposed into many simpler tasks each of which is allocated to the individual units of the array. The actuators are capable of mechanical feats far larger than their size would imply. For example, researchers have located small microactuators on the leading edge of airfoils of an aircraft and have been capable to steer it using only these miniaturised devices (5). 
Several physical principles have been exploited in distributed manipulation. Thermal expansion (6), electrostatic actuation (7) and electrostatic torsional resonators (8) are used in "cilia" arrays so called from their biological counterparts that can be found in the human respiratory tract. Through cilia's synchronous vibration small parts can be moved. Cilia arrays are an example of contact manipulation as they rely on friction for moving microparts. This can become an issue when dealing with fragile microparts or parts that cannot be contaminated. In microassembly contactless manipulation is a feasible alternative because of the small size and lightweight of the objects to be moved.

Contactless manipulation is advantageous as (9):

- Surface forces can be completely neglected

- It is suitable for handling fragile, freshly painted, sensitive micron-sized structured surfaces

- It allows the handling of non-rigid microparts

- There's no contamination of and from the end effector

Electrostatic actuation is used in (10) where particles are moved over an array of parallel field electrodes. The application of balanced multi phased voltages creates a travelling field-wave that conveys particles from electrode to electrode. In (11) a magnetic levitated wafer system transport system is developed for the semiconductor fabrication process to deal with the problem of oil contamination that normally exists in conventional transport systems. Contactless manipulation can also be realised using air jets to levitate and transport objects. In (12) an air paper mover is proposed as an alternative to motor-driven pinch rollers as physical contact can be undesirable for a variety of reasons. For instance, coated sheets can be damaged, sheets with toned images can have their images smeared or rollers can be contaminated by picking up coating material. Moreover, moving sheets along non straight trajectories is complex for cylindrical roller systems and the energy required to accelerate them is far greater than the energy effectively transferred to the paper. However this application is not suitable for microassembly as it is not able to provide a high level of precision.

\section{FOUR DIRECTIONS MICROACTUATOR}

A pneumatic contactless microfeeder based on the principle of distributed manipulation is proposed. The microfeeder consists of an array of micronozzles. Air is used for keeping the parts suspended. The parts are moved through the control of the micronozzles. As can be seen in figure 3, a 
single microactuator is made up of four nozzles formed by a central electrode and four walls around it.

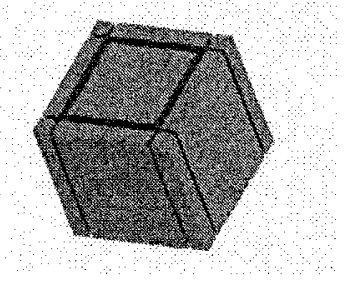

Figure 3. Pneumatic microactuator

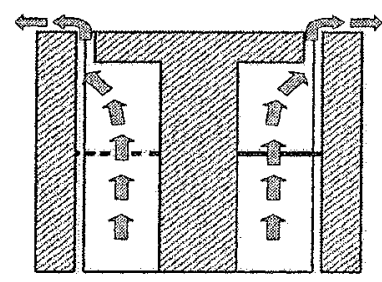

Figure 4. Cross section

The nozzles are opened or closed by electrostatic actuation. In the neutral position the four nozzles are all open (figure 5): the airflow, coming from the bottom of the microactuator, is equally divided among the four nozzles because of the symmetry of the structure. The outcoming airflows are such that the resulting force field causes the micropart to hover above the microactuator. For moving the object the central cursor is attracted towards one of the walls and the corresponding nozzle is closed. In figure 6 the rightwards and leftwards jets compensate each other hence there's a net force that pushes the micropart downwards. A similar working principle was presented in (13). The proposed design is advantageous because the microactuator is more compact as it keeps the dimensions of the airflow channel constant. Moreover, movement in four orthogonal directions is achieved with a single microactuator as opposed to the combination of four different microactuators capable of conveying objects in two directions only. This feature is of paramount importance as distributed manipulation becomes more effective if two conditions are satisfied: the microactuators have to be as small as possible as their size directly affects the minimum size of the parts that can be moved and the density of microactuators has to be high because this directly influences the position resolution that can be achieved. 


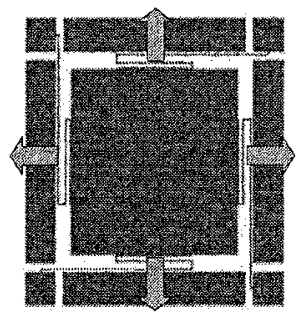

Figure 5. Top view of the microactuator in neutral position

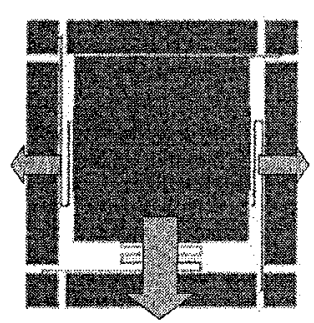

Figure 6. Top view of the microactuator when activated

The microfeeder manufacturing sequence is based on IC-compatible fabrication process so that it is possible to obtain a high number of microactuators all the same time. Details about the process are reported in chapter 4 . The array is then mounted and electrically connected to a printed circuit board. This means that the movable central electrode is fixed at its base and bends slightly for closing the nozzles. For this reason, as can be seen in figure 7, its lower part is connected with four springs to four "pillars" placed between the electrodes. The springs increase the robustness of the structure and help the electrode to return to its central position. This task can be accomplished also through the control of the electric field that acts upon the cursor.

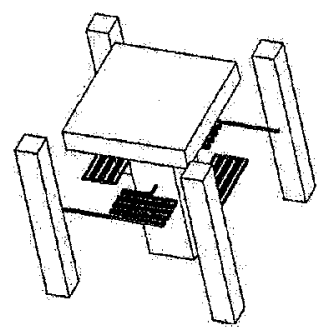

Figure 7. View of the microactuator without the side walls

\subsection{Microfeeding functionalities}

The air jets coming out of the microactuators form a force field that can be globally controlled through the local control of the nozzles. As a result of this coordinated motion several functionalities, which are specific tasks of a feeding system, can be generated. 


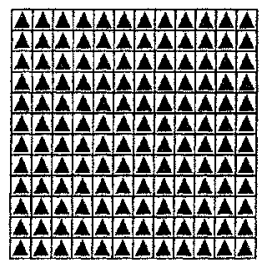

Figure 8. Transport mode

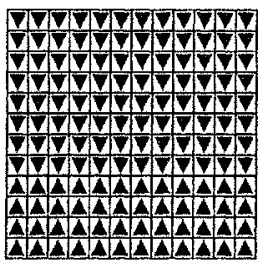

Figure 9. Aligning mode

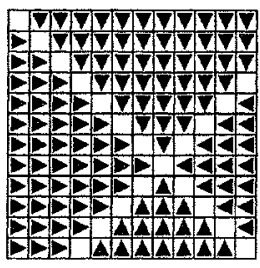

Figure10.

Positioning mode

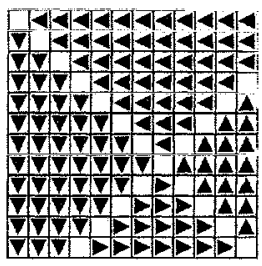

Figure 11. Rotating mode (14)

For transporting a part (figure 8), all the air jets point in the same direction. If there's the need to align microparts (figure 9), the feeder's surface can be divided into two regions in which the relative force fields move the parts into two opposite directions. The parts align along the border of the two regions. Airflows can be arranged in a way such that the microparts are moved to any specific position (figure 10). The borders between different regions with different force fields act as spatial filters. It is also possible to maintain the position and just change the orientation (figure 11) with four orthogonal force fields. As all the nozzles can be independently activated, the force field due to the air jets is dynamically controllable. Hence, several feeding functionalities can be obtained in cascade: a micropart placed on the feeder is moved to the desired position, its orientation is changed according to the particular needs and then it is moved to a different position which acts as a dead nest. The combination of feeding functionalities also offers the possibility of realising some elementary assembly operations as can be seen in figure 12: once the two microparts are in the positions $\mathrm{A}$ and $\mathrm{B}$, the force field is modified as represented. The combined microparts can be fed to a microassembly system as a subassembly thus simplifying the whole process.
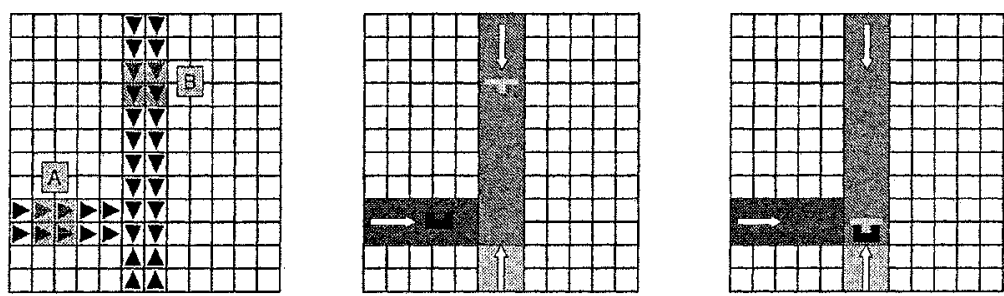

Figure 12. Combination of parts into a subassembly 


\section{MICROFEEDER FABRICATION PROCESS}

A manufacturing sequence that relies on batch fabrication techniques used in the IC industry was outlined. The reason behind such a choice is that, in this way, it is possible to obtain a large number of identical microactuators all at the same time. Figure 12 shows the steps of the fabrication process. A standard SOI (Silicon On Insulator) wafer is used with a $100 \mu \mathrm{m}$ top silicon layer, $4 \mu \mathrm{m}$ thick buried oxide layer and $525 \mu \mathrm{m}$ thick silicon base substrate. Before the actual process takes place, the substrate is thinned to a thickness of $250 \mu \mathrm{m}$ with a wet etching technique based on TMAH at $90^{\circ} \mathrm{C}$. During this step, the front side of the wafer is protected by a simple oxide layer of $50 \mathrm{~nm}$ realized by oxidation furnace deposition. In the first step of the fabrication process the lower silicon substrate is machined by deep RIE (Reactive Ion Etching). The material that is not going to be removed is protected with photo resist mask. RIE is chosen because, as an effect of its strong anisotropy, only the material perpendicular to the substrate surface is removed. As a result, high aspect ratio walls can be obtained. The next step is the fabrication of the springs that support the central cursor, again by RIE. In order to have the springs free to contract and expand, the oxide layer is removed by sacrificial layer (BOX) etching. HF (hydrofluoric acid) is used in this step as it helps to avoid sticking problems. After the releasing procedure, the top silicon layer is machined by RIE in order to obtain the clearance for the nozzles. 


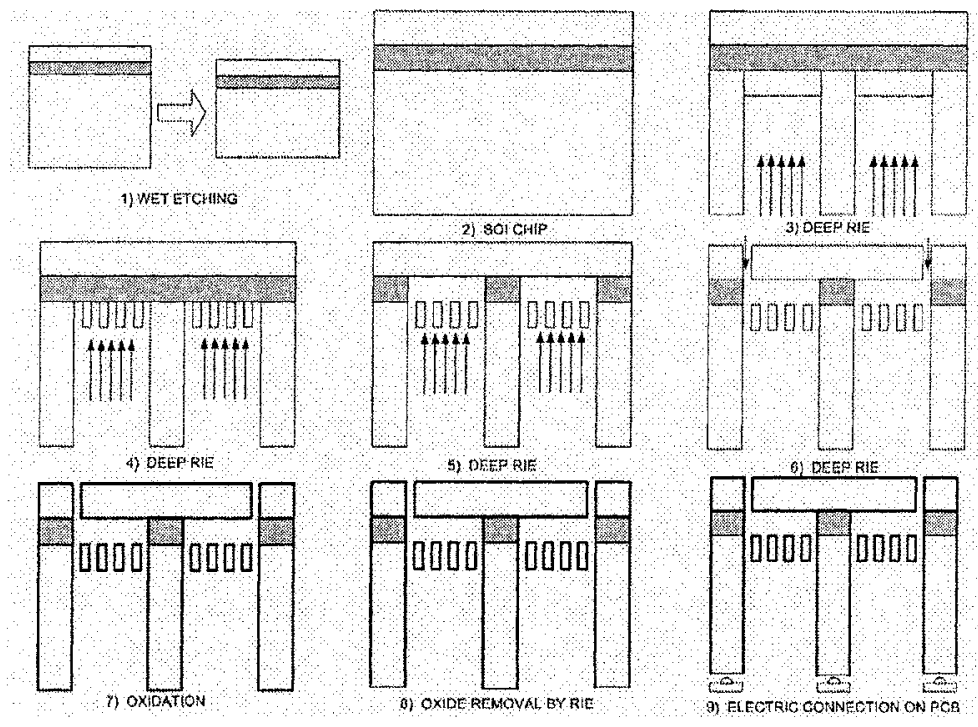

Figure 13. Fabrication process

The whole structure is then oxidised to ensure electrical insulation between the electrodes. In the next step RIE is used to remove the oxide from the bottom of the silicon substrate in order to allow the connectivity to the printed circuit board.

\section{CONCLUSION AND FUTURE WORK}

A pneumatic microfeeder for high precision assembly based on distributed manipulation was presented. Microparts float over an air cushion and are conveyed to the desired position with the desired orientation through the cooperation of individually controllable airflows. Further studies will be conducted to validate the proposed concept and evaluate its practical applicability using a prototype. For this purpose a manufacturing sequence based on IC-compatible fabrication process was outlined.

In a first stage, the validity of the proposed concept is going to be assessed using visual servoing to implement closed loop control. Ultimately, the IC-compatible fabrication process offers the opportunity to integrate on a single substrate electronic circuits, sensors and processing units. This is an important feature as the number of signals that have to be managed is significant because of the high density of actuators on the array. The integration of sensors and processing units grants each actuator a higher 
autonomy and reduces the amount of information exchange with the central processing unit; the execution of a motion task is realised through local information exchange among smart actuators. Results of these further investigations will be reported in due course

\section{REFERENCES}

1. Geng H., Semiconductor manufacturing handbook, Mc Graw-Hill Handbooks page 22.3

2. Alting L. et al. Micro Engineering, Annals of the CIRP Volume 52/2/2003

3. Koelemeijer Chollet S., Bourgeois F. and Jacot J., Economical justification of flexible microassembly cells, Proceedings of the $5^{\text {th }}$ IEEE International Symposium on Assembly and Task Planning, Besancon, France, July 10-11 2003 pages 48-53

4. Viinikainen H., Uusitalo J. and Tuokko R., New flexible minifeeder for miniature parts, Proceedings of the International Precision Assembly Seminar IPASS 2004, Bad Hofgastein, Austria, Feb 11-13, pages 87-94

5. Huang P. H. et al., Applications of MEMS devices to Delta Wing aircraft: from concept development to transonic flight test, AIAA paper No. 2001-0124, Reno, Nevada, January 8-112001

6. Ataka M. et al., Fabrication and operation of polyamide bimorph actuators for ciliary motion system, Journal of Microelectromechanical Systems, Dec 1993 2(4), pages 146150

7. Goosen J.F.L. and Wiffenbuttel R.F., Object positioning using a surface micromachined distributed system, Proceedings of the $8^{\text {th }}$ International Conference on Solid-State Sensors and Actuators and Eurosensors IX, Stockholm, Sweden, June 25-29 1995, Volume 2, pages 396-399

8. Böhringer K.F., Donald B.R., Mihailovich R. and MacDonald N.C., Sensorless manipulation using massively parallel microfabricated actuator arrays, Proceedings of the IEEE International Conference on Robotics and Automation (ICRA), May 1994, San Diego, California, USA, Volume 1, pages 826-833.

9. Lambert P., Vandaele V., Delchambre A., Non-contact handling in micro-assembly: state of the art, Proceedings of the International Precision Assembly Seminar IPASS 2004, Bad Hofgastein, Austria, Feb 11-13, pages 67-76

10. Moesner F. M. and Higuchi T., Contactless manipulation of microparts by electric field traps, Proceedings of the SPIE's International Symposium on Microrobotics and Microsystems Fabrication, Pittsburgh, October 1997, vol. 3202, pages 168-175

11. Park K. H., Lee S. K., Yi J. H., Kim S.H., Kwak Y.K. and Wang I.A., Contactless magnetically levitated silicon wafer transport system, Journal of Mechatronics $1996 \mathrm{Vol}$. 6 , No. 5, pages 591-610

12. Biegelsen D. Berlin A., Cheung P., Fromherz M., Goldberg D., Jackson W., Preas B., Reich J. and Swartz Lars, Airjet paper mover, presented at SPIE International Symposium on Micromachining and Microfabrication, 4176-11, Sept 2000

13. Fukuta Y., Yanada M., Ino A., Mita Y., Chapuis Y.A., Konishi S. and Fujita H., Conveyor for pneumatic two-dimensional manipulation realized by arrayed MEMS and its control, Journal of Robotics and Mechatronics, 2004, Volume 16, No. 2, pages 163170

14. Konishi S. and Fujita H., Two dimensional conveyance system using cooperative motions of many microactuators, Proceedings of IROS 96, Volume 2, pages 988-992 